Editorial

\title{
Non-model organism research in the changing genomic landscape
}

\author{
Monika Gulia-Nuss
}

Department of Biochemistry and Molecular Biology, University of Nevada, Reno, NV, USA

It is estimated that the planet earth is host to approximately ten million species of plants and animals with only approximately 1.5 million documented in the Catalogue of Life. However, our knowledge of biochemical, molecular, genetics, and cellular processes comes from the studies of fewer than a dozen organisms. Although focusing our research on these "model" organisms has paid off, the downside is that we know very little about the biology of the vast majority of organisms, the non-model organisms. Non-model organisms are organisms that have not been selected by the research community for extensive study mostly because they lack the features that make model organisms easy to investigate e.g. they cannot grow in the laboratory, have a long life cycle, low fecundity or poor genetics.

Keywords: Aedes aegypti, CRISPR, genome, Haemaphysalis longicornis, Ixodes scapularis, ticks

Received: 30 November, 2018, Accepted: 5 January, 2019.

Corresponding Author: Tel. 0017065936413, E-mail: mgulianuss@unr.edu

Print ISSN: 1687-7942, Online ISSN: 2090-2646, Vol. 12, No. 1, April, 2019.

With the advances in technologies such as next generation sequencing, RNA interference (RNAi); clustered, regularly interspaced short palindromic repeats (CRISPR) tools; and advanced imaging technologies, the timing is perfect for studying the diverse non-model organisms. Ticks, for instance, are fascinating organisms because they are almost indestructible; they survive years without food or water, freezing, and in case of some invasive species such as Haemaphysalis longicornis, even reproduce through parthenogenesis. These organisms with fascinating biology have been largely overlooked by the molecular and cell biologists mainly due to the above stated complexities.

In my laboratory, we are focusing on the tick, Ixodes scapularis, a major vector of Lyme disease in the United States. This is the only tick species for which genome has been sequenced and published. The I. scapularis genome $(2.1 \mathrm{Gbp})$ was sequenced using BAC clones and Sanger sequencing that has its own limitations in terms of inability to assemble the large, repetitive genome ${ }^{[1]}$. Advances in next generation sequencing technologies to achieve long reads for example Pacific Bio (Pac Bio), $10 \mathrm{X}$ genomics, and Oxford Nano pore are changing the sequencing landscape and now allow high quality reference assemblies for ticks to rival those produced for the mosquito vectors ${ }^{[2,3]}$. Aedes aegypti genome, another large, highly repetitive genome $(1.2$ Gbp) benefitted from the Hi-C scaffolding of assembled regions. This approach generated high quality reference assemblies for both the African malaria vector Anopheles gambiae and the yellow fever vector, Aedes aegypti ${ }^{[3,4]}$.

We successfully used Chicago and Hi-C assemblies for I. scapularis genome assemblies and scaffolding ${ }^{[5]}$.
In addition, complementary transcriptomic and proteomic studies are underway to validate predicted coding sequences and analyses of gene expression. A chromosome level genome assembly is the first step towards developing other state-of-art tools for studying tick vectors. With the availability of improved genome consisting of chromosome level scaffolds, we are now identifying tick-specific promoters that would allow the reporter expression for gene function studies using CRISPR tools. With our newly developed tick embryo injection technique in hand, we are in a strong position to carry out forward genetic approaches to identify genes associated with different aspects of tick biology such as vector competence, acaricide resistance, and new targets for a vaccine development.

In summary, the future of non-model genetics and genomics research is bright and we expect to see many laboratories investing their efforts in studying nonmodels so that we have a better understanding and appreciation of the biochemical, molecular, genetics, and cellular processes of diverse organisms.

\section{REFERENCES}

1. Gulia-Nuss M, Nuss AB, Meyer JM, Sonenshine DE, Roe RM, et al. Genomic insights into the Ixodes scapularis tick vector of Lyme disease. Nature Comm 2016; 7:10507.

2. Neafsey DE, Waterhouse RM, Abai MR, Aganezov SS, Alekseyev MA, Allen JE et al. Mosquito genomics: highly evolvable malaria vectors: the genomes of 16 Anopheles mosquitoes. Science 2015; 347(6217):1258522.

3. Matthews BJ, Dudchenko O, Kingan S, Koren S, Antoshechkin I, Crawford JE, et al. Improved reference genome of Aedes aegypti informs 
arbovirus vector control.. Nature 2018; 563(7732):501-507.

4. Miles A, Harding NJ, Bottà G, Clarkson CS, Antão $\mathrm{T}$, Kozak K, et al. Genetic diversity of the African malaria vector Anopheles gambiae. Nature 2017; 552(7683): 96-100.
5. Nuss AB, Sharma A, Gulia-Nuss M. Chicago and Dovetail Hi-C proximity ligation yield chromosome length scaffolds of Ixodes scapularis genome. BioRxiv. 2018; https://doi.org/10.1101/392126. Posted August 15, 2018. 